\title{
Force Attractor in Confined Comminution of Granular Materials
}

\author{
O. Ben-Nun, ${ }^{1}$ I. Einav, ${ }^{1}$ and A. Tordesillas ${ }^{2}$ \\ ${ }^{1}$ School of Civil Engineering, The University of Sydney, Sydney, New South Wales 2006, Australia \\ ${ }^{2}$ Department of Mathematics and Statistics, The University of Melbourne, Melbourne, Victoria 3010, Australia
}

(Received 12 October 2009; published 8 March 2010)

\begin{abstract}
We reveal a novel attractor in the space of contact forces that bounds the behavior of granular materials during confined comminution. The attractor is reached asymptotically as the porosity reduces and the grain size distribution attains an ultimate power law scaling. The ultimate distribution of the contact forces follows a clear log-normal distribution, distinctively different from previous observations in uncrushable systems. Supporting evidence comes both from comprehensive discrete element simulations and a theoretical Apollonian model.
\end{abstract}

PACS numbers: 81.05.Rm, 89.75.Da, 89.75.Fb

Systems with complex dynamics often have an attractor to which they evolve after long enough time [1]. Of particular relevance to this Letter are those systems that present transient dynamics (or "transient chaos" [2]) with a fixed-point attractor. Many of these attractors relate to the forces that drive the dynamics. Specifically, during the confinement of granular systems a fascinating set of force chains travels through the media [3]. The contact force distribution (CFD) is pivotal to understanding such matter [4-7], although these were for a limited degree of polydispersity and in the absence of grain crushing. Here, we investigate the evolution of the CFD in idealized 2D crushable granular materials. In this case the evolution of the CFD is inseparable from the evolution of grain sizes because crushing occurs in grains that are subjected to a critical combination of contact forces. Fragments rearrange to better fit in the available pores; subsequently, the local forces reorganize. We show that, in the space of the force chains [8], these interesting dynamics decay towards an ultimate condition. In particular, it is shown that the problem is highly sensitive to initial conditions. However, unlike trivial random systems, the current system is also shown to have an attractor with multiple fixed points, characterized by a unique CFD at the ultimate state.

The exact form of the CFD in jammed configurations of static granular assemblies was investigated by many, with Radjai et al. [4] proposing that the number of normal forces with a magnitude higher than the mean value decays as an exponential "tail." This tail was later found to have a steeper decay than initially thought $[5,6]$, particularly in sheared systems [7]. Back to our specific interest, the grain size distribution (GSD) evolves towards the smaller units: a process known as "confined comminution." Therefore, this problem presents a dynamical evolution in terms of the local forces, grain sizes, and the spatial configuration. A new way forward is to study the micromechanics of confined comminution via a dynamical systems approach. We take the first steps in this direction by examining the evolution of the force chains and the CFD in the context of attractors.
Model of confined comminution.-We employ a model of grain crushing based on the discrete element method (DEM) [9], which develops an ultimate power law GSD with a fractal self-similar pattern, in agreement with previous observations in granular physics and geosciences (soil, rock, and ice mechanics) [10]. This model employs the grain fracture criterion of [11] and the well established strategy of replacing a precrushed particle by postfracture fragments [12]. We calculate the major and minor principal representative forces acting on a given particle as follows:

$$
s_{1,2}=\left[\left(s_{x x}+s_{y y}\right) / 2\right] \pm \sqrt{\left[\left(s_{x x}-s_{y y}\right) / 2\right]^{2}+s_{x y} s_{y x}}
$$

where $s_{i j}=\sum_{c=1}^{N_{c}} n_{i}^{(c)} F_{j}^{(c)}(i, j=1,2), N_{c}$ is the grain's total number of contacts, the superscript $(c)$ stands for the $c$ th contact, and $n_{i}^{(c)}$ and $F_{j}^{(c)}$ are the components of the normalized branch vector and contact force vector, respectively [8]. The representative measures of the shear and the normal forces per particle are computed via $\Theta=$ $\left(s_{1}-s_{2}\right) / 2$ and $\Gamma=\left(s_{1}+s_{2}\right) / 2$. A particle of diameter $x$ crushes if a modified "Brazilian" criterion [11,13] of $2 \Theta-\Gamma \geq x \sigma_{f}$ is met; $\sigma_{f}$ denotes the particle's strength through $\quad \sigma_{f} \equiv \sigma_{f}(P)=\sigma_{f M} \ln (1 / P)^{1 / w}\left(x / x_{M}\right)^{-2 w}$. Following Jaeger [14], $\sigma_{f M}$ is the critical tensile stress for the failure of the biggest particle with a size $x_{M}$, the factor $\ln (1 / P)^{1 / w}$ captures Weibull's statistics of strengths [15], while $P \in[0,1]$ is a uniformly distributed variable and $w$ is Weibull's modulus.

The process of confined comminution is exemplified in Fig. 1. When a grain exceeds the failure criterion it is replaced by a set of three identical smaller fragments, inscribed within the circumference of the parent particle. This is achieved without introducing overlaps, and without limiting the number of fragment generations or their size. Mass is conserved by a rapid linear expansion of the fragments, while carefully avoiding excessive crushing from artificial overlaps [9]. More than 300 particles with normal and shear stiffness of $10^{3} \mathrm{MPa}(\mathrm{MN} / \mathrm{m}$ per $1 \mathrm{~m}$ thickness unit) are introduced into a rectangular loading chamber, 


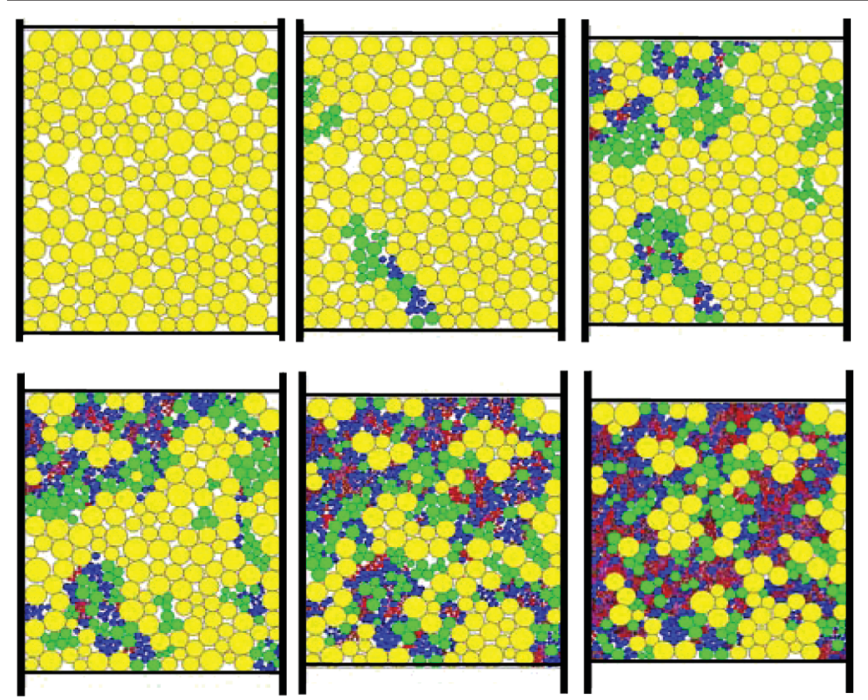

FIG. 1 (color). Results from the two-dimensional DEM model. Images (left to right, top to bottom) show the particles as they crush, from the first crushing event and at strain increments of $\Delta \varepsilon=0.03$ thereafter. Grain colors represent various fragment generations.

with normal and shear stiffness of $10^{5} \mathrm{MPa}$. The system consolidates to the jamming transition between the fluid and solid phases [16]. Motivated by experiments on brittle sand, particle density is set to $2000 \mathrm{~kg} / \mathrm{m}^{3}, w=4, \sigma_{f M}=$ $18 \mathrm{MPa}$, and the largest grain is taken $x_{M}=6 \mathrm{~mm}$. We set an initial uniform distribution of grain sizes by number, where the smallest initial grain size $x_{m}$ is set to give $x_{M} / x_{m}=2$ or 10 . The intergranular friction coefficient $\mu$ is chosen to be 0.6 while keeping the sidewalls fixed. The simulations follow for initial porosities of $\eta_{o}=\eta_{\max }$ or $\eta_{\min }$ (the maximum or minimum porosities for the jamming transition of the associated $x_{M} / x_{m}$ ). The axial load is increased gradually by bringing the upper and lower walls closer at a relative inwards velocity that grows slowly to $0.003 \mathrm{~m} / \mathrm{sec}$. The simulation is continued until an asymptotic limit is observed in each of the evolving GSD and CFD, with more than 11000 particles.

Figure 2(a) presents the porosity-pressure response curves as they develop in three simulations with varying index properties [9]. All curves evolve towards a single asymptotic line as the pressure increases. This is in agreement with many experimental observations [17]. This in fact may be seen as a motivation for attractors in such systems.

As the microscopic volume decreases, particles fracture and the GSD evolves. Specifically, the number of particles $N$ with a diameter $y$ greater than $x$ approaches asymptotically an ultimate power law set, i.e., $N(y>x) \propto x^{-\alpha}$, with $\alpha=1.3$ [see Fig. 2(b)], where particles are so confined that the relative displacements become affine. As the number of interactions goes to infinity, the system is expected to develop a complete fractal set. The corresponding evolution of the CFD is shown in Figs. 2(c) and 2(d). Before

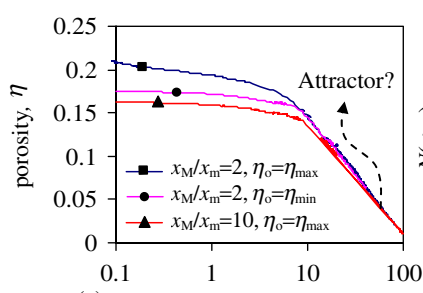

(a) pressure $(\mathrm{MPa})$
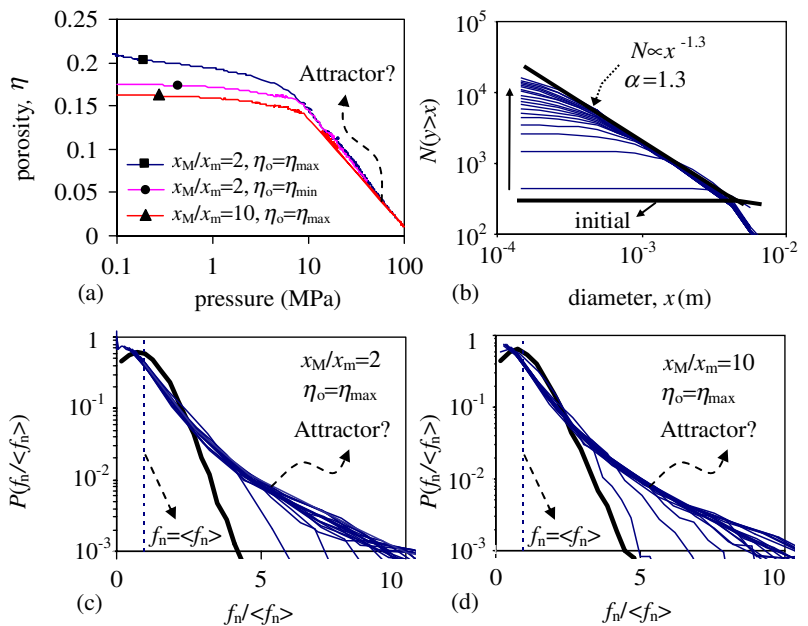

(b) diameter, $x(\mathrm{~m})$

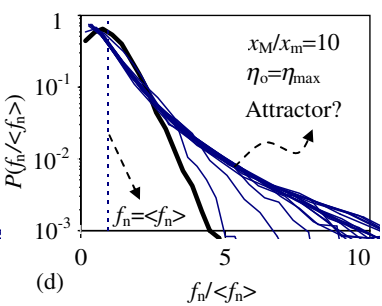

FIG. 2 (color online). (a) Porosity-pressure curves for various initial porosities $\eta_{o}$ and initial GSD by changing $x_{M} / x_{m}$; (b) typical evolution of GSD from initially uniform to an ultimate power law, plotted at every pressure increment of $5 \mathrm{MPa}\left(x_{M} / x_{m}=2\right.$ and $\left.\eta_{o}=\eta_{\min }\right) ;(\mathrm{c})$,(d) typical evolutions of the CFD. Note that the final CFDs are shown again in Fig. 5, with prediction.

crushing, the CFD has an exponential tail, but with a steeper decay than that proposed in [4], in agreement with $[5,6]$. However, the CFD changes markedly during the confined comminution, in a clear deviation from either propositions of [4] or [5,6]. In particular, as the CFD evolves, another asymptote is revealed: an ultimate CFD that is independent of the initial porosity and GSD (as amplified later in Fig. 5).

Force chains dynamics. - To resist external loads granular materials transmit forces through the contact force network. However, only a part of this network carries the majority of the load. We adopt the algorithm in [8] and show only force chain particles. To belong to a force chain, a particle must satisfy two requirements. First, the magnitude of its representative major principal force, $s_{1}$ [Eq. (1)], has to be greater than the overall average of the sample $\left\langle s_{1}\right\rangle$. Second, the direction of $s_{1}$ must be quasilinear with the direction of the $s_{1}$ of a neighboring particle, which must also satisfy the first magnitude criterion. "Quasilinear" refers to a change in directions of less than $45^{\circ}$. Here, the magnitude criterion is slightly different than that originally proposed by [8]: instead of an average $s_{1}$ by number, we use a mass average $\left\langle s_{1}\right\rangle=\left(M_{T}\right)^{-1} \sum_{k=1}^{N} s_{1}^{(k)} m^{(k)}$, which is more useful for visualizing the bulk picture. $N$ is the total number of particles in the sample, that varies during loading, and $s_{1}^{(k)}$ and $m^{(k)}$ are the magnitude of the representative major principal force and mass of particle $k$, respectively.

Figure 3 presents the force chains during the simulation of two nearly identical samples, with an initial $x_{M} / x_{m}=2$ and $\eta_{o}=\eta_{\max }$. The sole difference is made simply by deleting a single particle (the strength parameters are deterministically similar). The first images of the force 

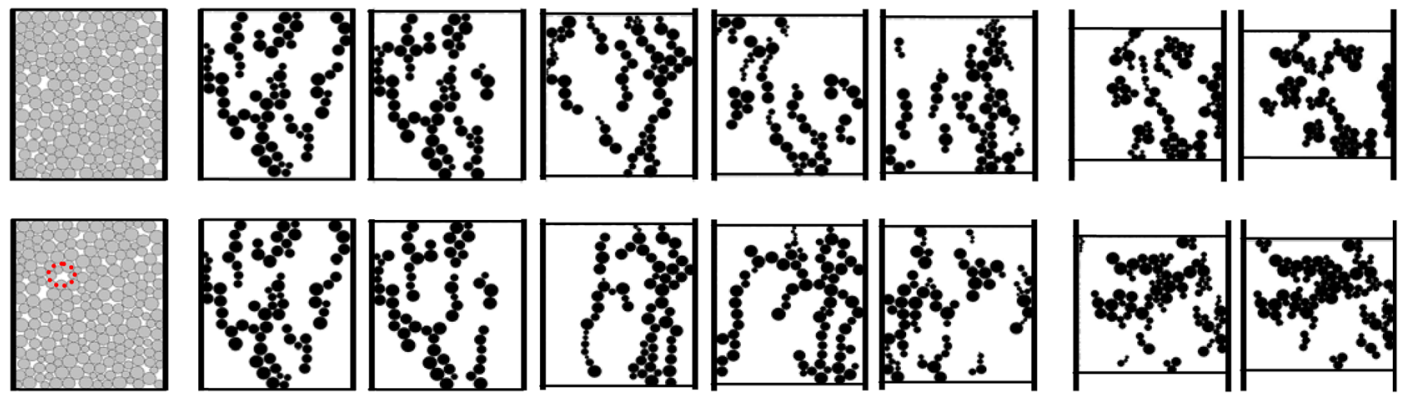

FIG. 3 (color online). Sensitivity of force chain evolution to minor changes in initial conditions. Images from left, top and bottom: initial samples at the jamming transition (strain $\varepsilon=0.0$ ) in two nearly identical simulations, differing only by a single omitted particle (marked by the dotted circle in the bottom left image); force chains in the two samples, first at every strain increment of $\Delta \varepsilon=0.02$ from $\varepsilon=0.0$ to $\varepsilon=0.08$, and then at $\varepsilon=0.2$ and 0.3 . Note the increasing disparity in the two force chain networks-to the extent that traces of similarities in the force chain topologies at $\varepsilon=0.0$ become undetectable: see last two states in which markedly different ultimate topologies develop.

chains are almost identical for the two samples, and correspond to the respective jamming transition. In the early stages after the jamming transition (e.g., $\varepsilon=0.02,0.04$ ), small disparities in the force chain network of the two samples emerge whenever a particle crushes. Thus, one may conceive that the two systems originate from a nearly identical force chain network at the jamming transition. However, as the simulations proceed (e.g., $\varepsilon=0.06,0.08$ ), the force chain topologies deviate considerably. Finally, as the GSD evolves towards the ultimate power law [Fig. 2(b) ] the transient dynamics of the force chains, driven by grain crushing, decay asymptotically towards a fixed point (e.g., last two states $\varepsilon=0.2,0.3)$. On the one hand, we have smooth macroscopic porosity-pressure curves [Fig. 2(b)] that merge towards a unique energy state; on the other hand, we have a complex transient dynamics that eventually freezes in a deterministic force chain configuration, but that is highly sensitive to minute changes in initial conditions. However, as we shall see in the following, this does not affect the contact force distribution, which is unique and can be predicted using the following simple model. Therefore, the current system has multiple fixedpoint attractors in the space of the force chains.

Simple Apollonian model.- Recall that, in the crushable DEM simulations, the number $N$ of particles with a diameter $y$ greater than $x$ develops towards an ultimate power law set $N(y>x) \propto x^{-\alpha}, \alpha=1.3$. This coefficient is strikingly similar to the fractal dimension of an Apollonian gasket with 25 generations, $\alpha=1.305684$ [18], where the porosity is essentially zero. These similarities in fractal dimensions and in porosities motivate our next analysis using a simple Apollonian model. We consider an Apollonian packing with a friction coefficient $\mu=0$ and 0.6; Fig. 4. The entire gasket is then subjected to a slow but minimal isotropic compaction from the exterior, which is damped towards the static state. We then extract the contact forces. The CFDs at the end of these simple simulations are plotted in Fig. 5(b); compare these with the final CFDs of the comprehensive crushable DEM simulations in Fig. 5 (a). Strikingly, all of the probabilities can be described by a single unique log-normal distribution (see continuous lines in Fig. 5):

$$
p\left(f_{n}^{*}\right)=\frac{1}{f_{n}^{*} \sigma \sqrt{2 \pi}} \exp \left[-\frac{1}{2 \sigma^{2}}\left(\ln \frac{f_{n}^{*}}{\Omega}\right)^{2}\right],
$$

where $f_{n}^{*}=f_{n} /\left\langle f_{n}\right\rangle, \sigma$ is the standard deviation of the variable's natural logarithm, and $\Omega$ is a scale parameter that is found to be equal to 0.5 . We highlight the distinct contrast of this result with previous observations of CFD. Those dealt with uncrushable granular media, where the prescribed grain sizes were limited in the degree of polydispersity [4-6]. In particular, the insets in Fig. 5 focus on the tail of the ultimate distribution using a triple-log plot proposed by [6]: previous works show distributions with a slope between 1 and 2 [6]; here the slope is about 0.5. Also note that the Apollonian model was constructed using 10 generations, giving a fractal dimension $\alpha \approx 1.24$ over two decades, which can explain the deviation from the lognormal fit.

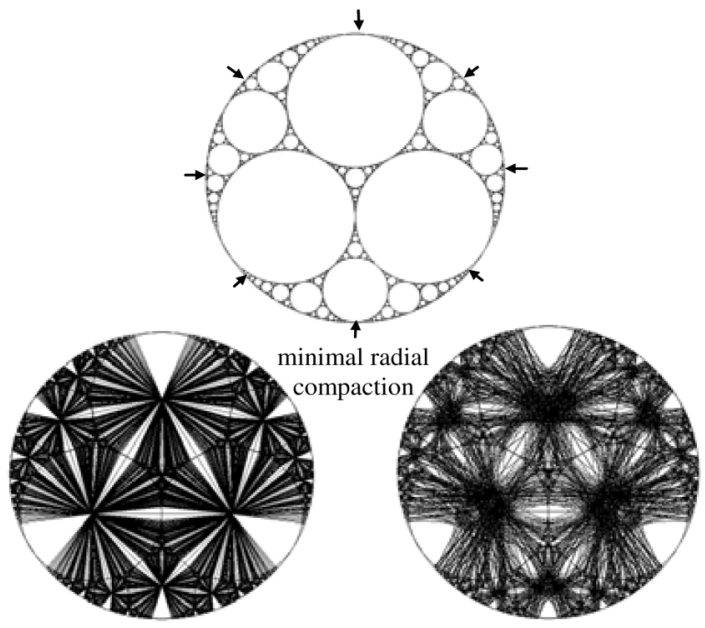

FIG. 4. Radial compression of an Apollonian gasket. Upper plot shows the particles as they are subjected to a minimal radial compression. Left-hand and right-hand images show the contact force network for $\mu=0.0$ and $\mu=0.6$, respectively. 

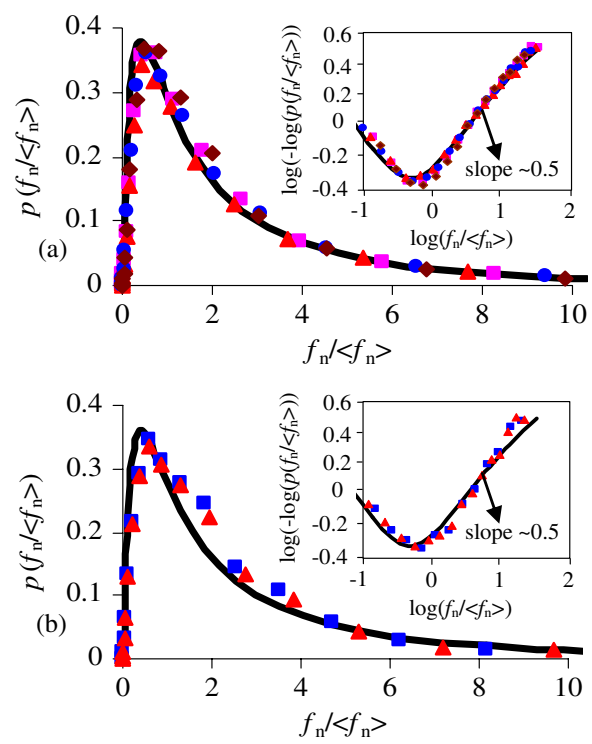

FIG. 5 (color online). The ultimate CFD. (a) From the comprehensive crushable discrete element simulations: $\boldsymbol{\Delta} d_{M} / d_{m}=2$ and $\eta_{o}=\eta_{\min }, \quad d_{M} / d_{m}=10$ and $\eta_{o}=$ $\eta_{\max }, \mathbf{\square}$ and $\backslash$ diamondsuit $d_{M} / d_{m}=2$ and $\eta_{o}=\eta_{\max }$ corresponding to Figs. 3(a) and 3(b). (b) Predicted by the simple Apollonian model: $\boldsymbol{\Delta} \mu=0.6$ and $\boldsymbol{\square} \mu=0.0$. Insets show the triple-log plot. The solid lines show the theoretical log-normal distribution with $\Omega=0.5$.

In summary, we have investigated the evolution of the CFD and of force chains based on a crushable DEM model and an Apollonian model. We reveal the presence of attractors. The system presents complex transient dynamics that are characterized by the high sensitivity of the evolution of the force chain topology to small changes in initial conditions. Unlike the sensitivity of the ultimate topology, the ultimate force distribution is insensitive to those small changes (Fig. 5). This suggests that our crushable granular material has multiple fixed-point attractors in the space of the contact forces, with each ultimate topology related to a single fixed point.

Without comminution, during confinement the normalized CFD in granular systems does not vastly change after the jamming transition. This is well known, and was documented in previous studies [5]. For confined comminution, the ultimate fractal GSD to which the DEM model evolves in the long term, a property shared by the Apollonian gasket, leads to an ultimate CFD that is described by a unique log-normal distribution. Clearly, it is the particular choice of (nominally zero) porosity and the (power law) GSD that determines the shape of the CFD. Thus, confined comminution is simply the mechanical route to reduce porosity by shifting the GSD towards a desirable ultimate power law, with which the force attractor could be reached. Future studies will map out the full dynamics of CFD and GSD from phase portraits of the confined comminution system for experimentally relevant parameters.
O.B.N. gratefully acknowledges the support of the School of Civil Engineering at The University of Sydney. I.E. and A.T. acknowledge their ARC Grant No. DP0986876. Finally, the authors wish to thank Thomas Stemler and Raphael Blumenfeld for the fruitful discussions, and the very useful comments by the anonymous reviewers that helped improve the Letter.

[1] E. N. Lorentz, J. Atmos. Sci. 20, 130 (1963); D. Ruelle and F. Takens, Commun. Math. Phys. 23, 343 (1971).

[2] S. Collin, The Lorenz Equations: Bifurcations, Chaos, and Strange Attractors (Springer, New York, 1982).

[3] A. Drescher and G. de Josselin de Jong, J. Mech. Phys. Solids 20, 337 (1972).

[4] F. Radjai, M. Jean, J.-J. Moreau, and S. Roux, Phys. Rev. Lett. 77, 274 (1996).

[5] L. E. Silbert, G. S. Grest, and J. W. Landry, Phys. Rev. E 66, 061303 (2002); J. Brujić, S. F. Edwards, I. Hopkinson, and H. A. Makse, Physica (Amsterdam) 327A, 201 (2003); K. Bagi, Granular Matter 5, 45 (2003); B. P. Tighe, A. R. T. van Eerd, and T. J. H. Vlugt, Phys. Rev. Lett. 100, 238001 (2008).

[6] A. R. T. van Eerd, W. G. Ellenbroek, M. van Hecke, J. H. Snoeijer, and T.J.H. Vlugt, Phys. Rev. E 75, 060302 (2007).

[7] E. I. Corwin, H.M. Jaeger, and S.R. Nagel, Nature (London) 435, 1075 (2005); T. S. Majmudar and R.P. Behringer, Nature (London) 435, 1079 (2005).

[8] J.F. Peters, M. Muthuswamy, J. Wibowo, and A. Tordesillas, Phys. Rev. E 72, 041307 (2005).

[9] O. Ben-Nun and I. Einav, Phil. Trans. R. Soc. A 368, 231 (2010).

[10] D. L. Turcotte, J. Geophys. Res. 91, 1921 (1986); C. G. Sammis, G. King, and R. Biegel, Pure Appl. Geophys. 125, 777 (1987); A. C. Palmer and T. J. O. Sanderson, Proc. R. Soc. A 433, 469 (1991); Y. P. Cheng, M. D. Bolton, and Y. Nakata, Geotechnique 54, 131 (2003); I. Einav, J. Mech. Phys. Solids 55, 1274 (2007).

[11] O. Tsoungui, D. Vallet, and J.C. Charmet, Powder Technol. 105, 190 (1999).

[12] J. A. Astrom and H. J. Herrmann, Eur. Phys. J. B 5551 (1998).

[13] E. M. R. Fairbairn and F.-J. Ulm, Mater. Struct. 35, 195 (2002); S. P. Timoshenko and J. N. Goodier, Theory of Elasticity (McGraw-Hill, New York, 1970).

[14] J. C. Jaeger, Int. J. Rock. Mech. Min. Sci. 4, 219 (1967).

[15] W. Weibull, J. Appl. Mech. 18, 293 (1951).

[16] A. J. Liu and S. R. Nagel, Nature (London) 396, 21 (1998); E. Aharonov and D. Sparks, Phys. Rev. E 60, 6890 (1999); H. A. Makse, D. L Johnson, and L. M. Schwartz, Phys. Rev. Lett. 84, 4160 (2000).

[17] J. A. Yamamuro, P. A. Bopp, and P. V. Lade, J. Geotech. Eng. 122, 147 (1996); Y. Nakata, Y. Kato, M. Hyodo, A.F. L. Hyde, and M. Murata, Soils Found. 41, 39 (2001); G. R. McDowell and C.M. Daniell, Géotechnique 51, 173 (2001).

[18] S.S. Manna and H. J. Herrmann, J. Phys. A 24, L481 (1991); R. D. Maudlin and M. Urbanski, Adv. Math. 136, 26 (1998). 